\title{
MEMORANDA
}

\section{Algunas reflexiones en torno a la salud de comunidades indígenas}

Piñeros M. ${ }^{1}$

\section{Problemática}

La población indígena en Colombia asciende a aproximadamente 600.000 individuos, repartidos en 87 etnias diferentes, que a pesar de ser distintas entre sí, tienen en común su origen amerindio y constituyen un sector social definido.

La mayor parte de la población indígena del país habita áreas de muy baja densidad poblacional y, de acuerdo con su localización geográfica, el Departamento Nacional de Planeación los ha clasificado como habitantes de cuatro macroregiones, a saber: selva, sabana, región andina y península guajira.

A pesar de pertenecer a tan diverso número de etnias y de habitar en regiones con características ecológicas tan distintas que influyen en el comportamiento epidemiológico de las enfermedades, se observa, en términos generales, una alta frecuencia de enfermedades infecciosas respiratorias y gastrointestinales, así como enfermedades transmisibles, desnutrición, problemas perinatales y patologías orales.

Aunque se desconoce la real magnitud de dichos problemas, en general, podemos afirmar que la situación de salud en las comunidades indígenas es crítica y preocupante.

Se mencionan a continuación una serie de factores y circunstancias que inciden o están relacionados con esta crítica situación y que podemos generalizar para la gran mayoría de comunidades indígenas del país.

\section{Situación de contacto y cambio cultural}

Antes de establecer contacto con la sociedad occidental, la forma de vida de la mayor parte de los indígenas compartía unas características generales como eran una menor densidad de población, un relativo aislamiento tanto cultural como geográfico, una mayor movilidad, un estrecho contacto con la naturaleza con una menor degradación del ecosistema y unas normas culturales muy estrictas.

Al entrar en contacto con la sociedad mayor se han ido alterando estas características, en mayor o menor grado, con las consiguientes consecuencias negativas sobre la salud de los individuos

Por un lado, han entrado en contacto con una serie de enfermedades desconocidas, principalmente de tipo viral, que desencadenan o desencadenaron epidemias severas -en ocasiones devastadorasdebido a la baja respuesta inmunológica a enfermedades nuevas y a las características especiales que tiene una epidemia cuando se presenta en un grupo de éstos: al afectar a un gran número de individuos simultáneamente, no hay quien pueda suministrar dentro de un mismo núcleo familiar cuidados generales, atención y alimentación, agravando así el curso y pronóstico de la enfermedad.

Por otro lado, el contacto por lo general ha conllevado a una pérdida de creencias, tabúes y prácticas como es el caso del infanticidio y de prácticas de espaciamiento entre los hijos que, al perderse, tienen claras consecuencias sobre la densidad de la población, incrementándola.

1 Investigadora científica. División de Investigación Social en Salud, Instituto Nacional de Salud, Santafé de Bogotá. 
La asimilación acelerada de prácticas y elementos externos generalmente genera estrés y comportamientos psicopatológicos al perder elementos conciliadores de la propia cultura. Son numerosos los ejemplos de suicidios y postración prolongada en grupos indígenas forzados a la sedentarización. Además, se asimilan muy frecuentemente hábitos negativos como el tabaquismo y el alcoholismo, que, al no acompañarse de la asimilación de elementos reguladores de la misma cultura, terminan en conductas perfectamente patológicas.

Al perder elementos culturales propios se va perdiendo la identidad y la confianza como grupo, así como el sentido de pertenencia a éste. A este proceso han contribuido históricamente, la persecusión y el desprecio por los recursos humanos y creencias propias, sobre todo en lo referente a prácticas de salud, dados por la evangelización y, en parte también, por la medicina occidental.

Otra de las consecuencias del contacto, es el paso de una economía de subsistencia a una economía de mercado, lo cual tiene graves consecuencias sobre la integridad del medio ambiente, ya que los ingresos provienen del comercio con bienes extraídos del medio, deteriorándolo paulatinamente. El deterioro del medio y el paso a una economía de mercado inciden negativamente en el nivel de nutrición con una ingesta cada vez menor de proteínas de origen animal y la adquisición en el mercado de elementos de bajo valor nutricional. Los problemas nutricionales suelen ser de los primeros y principales que aparecen cuando una sociedad va perdiendo su cultura tradicional.

Otra serie de cambios han sido determinados por la disminución de la movilidad y la sedentarización que suelen observarse como producto del contacto interétnico.

La sedentarización y otros factores mencionados anteriormente, así como la reducción del territorio, producen un aumento en la densidad de la población, que a su vez conlleva dificultades en la consecución de alimentos por reducción del territorio tradicional, lo que se traduce en problemas nutricionales. Además, aparecen problemas de hacinamiento y vivienda, problemas de disposición de excretas y basuras y degradación progresiva del medio ambiente, con el consiguiente incremento en la transmisión de enfermedades infecciosas y parasitarias y el aumento de vectores transmisores de enfermedades, tornándose en un círculo vicioso.

Son precisamente este tipo de problemas de salud los que vemos en la mayoría de las comunidades indígenas: desnutrición, infecciones parasitarias, tuberculosis, etc.

Podemos afirmar que entre menos aculturado esté un grupo indígena y menos dependencia tenga de la sociedad no indígena, mejor es su estado de salud.

\section{Prácticas tradicionales}

Dentro de su forma de vida y cultura los indígenas desarrollaron todo un sistema de prácticas y pensamientos favorables a la salud, sistemas que fueron válidos durante muchísimos años y aún siguen vigentes en muchas comunidades auncuando con menos fuerza, claridad e impacto, debido al contacto con la sociedad no indígena. Dentro de estas prácticas favorables podemos mencionar, por ejemplo, los largos períodos de lactancia materna, el espaciamiento entre embarazos, el uso de las plantas masticables para la prevención de caries dentales y la alta movilidad.

Para los indígenas, la enfermedad va más allá de lo orgánico y nuestro sistema biomédico no es un sistema explicativo válido para ellos. Al interior del universo sociocultural particular, cada cultura construye un sistema propio de clasificación, etiología, diagnóstico, tratamiento y pronóstico de las enfermedades.

Los sistemas médicos tradicionales se han ido debilitando porque no encuentran modelos explicativos y tratamientos para gran parte de las nuevas patologías, producto del contacto, que afectan a estas comunidades. Es así como existe una real dependencia del sistema médico occidental, para resolver muchos de los problemas que los aquejan, lo cual a su vez los coloca en una situación de ambivalencia. 


\section{Interculturalidad}

Del mismo modo como la religión, el lenguaje y los sistemas de parentesco constituyen sistemas culturales definidos, así también podemos ver la medicina como un sistema cultural con significados simbólicos que hacen parte de toda una realidad social que se construye desde la infancia, internalizando las normas que gobiernan su comportamiento, sus relaciones interpersonales y su percepción y entendimiento del mundo tanto interno como externo.

Salud, enfermedad, curanderos, doctores y pacientes, forman todos parte de este sistema y constituyen una importante fuente de estudio para entender cómo se configura culturalmente un sistema dado. Así mismo, también están determinados culturalmente la forma y manera de reaccionar de la gente, el uso de los distintos componentes, la concepción de la enfermedad, la denominación, las causas que se le atribuyen, la manera como se explica y se trata la enfermedad.

Cuando se presta atención en salud a grupos indígenas, se trabaja en un ámbito de interculturalidad donde se contraponen conceptos y creencias de dos universos y sistemas explicativos completamente distintos, lo cual plantea serias dificultades en varios aspectos.

Aparecen toda una gama de situaciones en las cuales interfieren las creencias con sus implicaciones, como son el dejarse examinar, el sexo del médico, el ámbito hospitalario, el tipo de droga que se receta, la no aceptación de tratamientos, etc.

A raíz del contacto con la sociedad occidental, las comunidades indígenas están experimentando una serie de cambios en sus formas sociales tradicionales los cuales, obviamente, tienen profundas implicaciones en el sistema cultural salud-enfermedad. Nos encontramos, entonces, ante realidades sociales que combinan creencias, valores e instituciones de ambos sistemas culturales en diferentes procesos y grados que pueden variar desde asimilación, complementariedad y conflicto hasta francas contradicciones.

\section{Accesibilidad y prestación de servicios de salud}

La mayor parte de las comunidades indígenas habitan zonas de difícil acceso, con largas y usualmente costosas jornadas entre una y otra comunidad.

A este problema de accesibilidad física, se suma generalmente el hecho de vivir en regiones donde actualmente hay severos problemas de orden público y cultivos ilícitos (como la coca y la amapola), generando situaciones de violencia cotidiana y dificultades para la movilización, condiciones que determinan para estas zonas un recurso humano en salud muy escaso.

Con respecto a la prestación de servicios de salud del sector oficial, muchas comunidades cuentan con un promotor de salud (el cual debe ser indígena) entrenado por personal de los servicios de salud. Es conocida la problemática en torno a los promoiores indígenas de salud, cuya capacitación no parece ser del todo acorde con las funciones que deben desempeñar, quienes reciben muy poca supervisión y asesoría, una dotación generalmente insuficiente, a quienes toca asumir prácticamente toda la atención en salud de los enfermos que se presentan en la comunidad y que presentan un alto porcentaje de deserción.

El poco personal médico que trabaja en estas zonas, generalmente está realizando su año rural y no tiene, por tanto, experiencia en el trabajo con comunidades indígenas. En general, cuando las comunidades son visitadas por el médico, lo cual sucede muy pocas veces al año, la atención que se presta es de afán, basada en un diagnóstico clínico que no es corroborado por laboratorio y descuidando muchos aspectos como la salud oral, donde la falta de unidades móviles de odontología no permite una atención curativa sino mutilante. 
Este tipo de atención, obviamente no puede tener mayor impacto sobre el estado de salud de los individuos y plantea el interrogante de si realmente vale la pena continuar con este tipo de atención donde, además de los problemas de baja cobertura y de acceso, muchas veces también hay baja utilización de los servicos por razones culturales.

\section{Falta de información}

Aunque sabemos cuales son las principales patologías que afectan a las comunidades indígenas en general, desconocemos la verdadera magnitud del problema ya que carecemos de estudios epidemiológicos e investigaciones serias al respecto.

En general, la falta de información que se tiene respecto al estado de salud de las comunidades indígenas, así como el problema del subregistro, pueden atribuirse a varios factores:

1. Por un lado, los registros sólo dan información de personas que acuden a los centros asistenciales, lo cual, teniendo en cuenta los problemas de accesibilidad tanto física como cultural, corresponde a un porcentaje muy bajo de la población indígena. Vale la pena anotar que la OMS ha estimado la medicina tradicional como el primer recurso disponible para el $80 \%$ de la población en áreas rurales. Además, no siempre la medicina alopática (aún cuando está disponible) constituye la primera elección y gran parte de los padecimientos o enfermedades son solucionados en el hogar o se acude a otro tipo de personal (curanderos, chamanes, etc.) para solucionarlos.

De acuerdo con las instrucciones del sistema nacional de información para el análisis, de morbilidad solamente se incorporan los diagnósticos realizados por médicos; los datos que se obtienen a nivel local corresponden a comunidades donde hay promotores o auxiliares, ilustrando claramente el problema del subregistro.

2. Suele haber dificultades logísticas en la remisión de registros o de consolidados desde el nivel local al nivel regional, conllevando muchas veces a la pérdida o confusión de los datos.

3. En los distintos formularios del subsistema de información, no se consigna el grupo étnico al cual pertenecen los individuos, de manera que cuando los datos son analizados en el nivel regional, es imposible obtener datos que se refieran exclusivamente a los distintos grupos étnicos.

4. Tanto a nivel comunitario como en el caso de los promotores, quienes son los encargados de recoger la información, posiblemente tampoco haya claridad con respecto a la importancia que tiene en un momento dado, el hecho de consignar algunos datos para poder analizarlos, sacar conclusiones y tomar decisiones. La información no se utiliza para la evaluación a nivel local y los formularios no son del todo adecuados para hacer seguimientos de coberturas de vacunación, empleo de medicamentos y de conductas del promotor.

5. Finalmente, la información que se consigna, así como los indicadores usados para establecer los estados de salud, tampoco contemplan categorías clasificatorias culturalmente aceptables que integren la interpretación y visión cultural de las comunidades objeto. Esta es solamente una de las manifestaciones de la gran diferencia conceptual que existe entre dos culturas que se encuentran en un momento dado. Al respecto, vale la pena mencionar un estudio realizado en hospitales y centros de atención psiquiátrica en Santafé de Bogotá, donde un porcentaje considerable de pacientes de clase popular afirmaron enfáticamente, estar embrujados y no locos, afirmación que otros pacientes reconocían y validaban. Un promotor perteneciente a esta cultura pensará que están embrujados pero muy seguramente omitirá la consignación de este tipo de patologías culturales, o si la consigna, esta información no será tenida en cuenta e irá a engrosar el ya saturado listado de síntomas y estados morbosos mal definidos. 
Además de no contemplar este tipo de categorías, tampoco se obtiene información acerca de recursos tradicionales que prestan atención en salud.

\section{Recomendaciones}

Plantear soluciones definitivas a un problema tan complejo es supremamente difícil; sin embargo, se mencionarán algunos puntos importantes a tener en cuenta en la búsqueda de soluciones.

Auncuando los distintos grupos indígenas se encuentran en momentos históricos con grados de aculturación muy diferentes entre sí, para efectos de las recomendaciones, es importante considerarlos como un sector social definido, para el cual hay que desarrollar y poner en práctica unos lineamientos generales de trabajo que contemplen su particularidad sociocultural.

Como primera medida, hay que reconocer la diversidad étnica y cultural para poder crear, en los diferentes niveles, el respeto hacia las comunidades indígenas y poder considerar la importancia que tienen en términos de patrimonio cultural.

Esta conciencia es importante para que haya una determinación política con respecto a la necesidad de mejorar la atención en salud para las comunidades indígenas, adecuarla a sus características y garantizar la asignación de recursos y presupuesto específico, tanto a nivel central como local. Esta asignación de recursos adquiere una mayor dimensión, si se tiene en cuenta el derecho a la atención gratuita que tienen los indígenas.

Hay consenso general acerca de la importancia de difundir y cumplir en los distintos niveles el decreto 1811 de 1990, particularmente con respecto al artículo octavo que ordena crear a nivel central en el Ministerio de Salud, un grupo de atención a comunidades indígenas que pueda definir las políticas generales, prestar asesoría a los distintos servicios seccionales de salud y, en general, coordinar las diversas accciones.

Para que este grupo pueda tener algún impacto, se necesita a nivel regional una persona o grupo responsable dedicado a coordinar el trabajo con las comunidades y servir como interlocutor principal entre el servicio y las comunidades. Esto nos lleva a otra de las principales recomendaciones que es la del diálogo y la confianza que deben buscar aquéllos que trabajan con comunidades indígenas y, particularmente, los trabajadores del área de la salud.

Con respecto a la prestación de servicios, es necesario que el recurso humano que trabaja con comunidades indígenas tenga un conocimiento previo de los elementos culturales, de la forma de vida y del sistema médico prevalente. Si se pretenden realizar acciones de educación en salud con indígenas, hay que tener en cuenta y conocer las creencias y actitudes que puedan incidir en el proceso salud-enfermedad. Esto se puede ilustrar, por ejemplo, con la dificultad para que ciertas culturas hiervan el agua de consumo, ya que el agua hervida representa algo caliente que está contraindicado en muchas situaciones de la vida diaria.

Es importante que se puedan rescatar y estimular aquellas prácticas propias de la medicina indígena que son favorables para la salud y realizar actividades educativas en relación a otras prácticas nocivas. Las prácticas tradicionales siguen cumpliendo un papel fundamental en muchas comunidades, lo cual hay que recordar y tener en cuenta para llegar con una actitud abierta, sin prejuicio, hacia estas tradiciones.

Dentro de los trabajos prioritarios por hacer, está el de diseñar modelos de información epidemiológica que permitan establecer el estado de morbimortalidad de las distintas comunidades y etnias. Esto implica que igualmente habrá que rediseñar la papelería que llevan los promotores indígenas, además de analizar la información que se registra. 
Igualmente, se deberán desarrollar procesos investigativos que busquen comprender los sistemas explicativos del proceso salud-enfermedad al interior de la cultura y procuren la adecuación de la prestación de los servicios de salud.

Dadas las circunstancias especiales en que se encuentran los promotores de salud, pareciera viable recomendar un entrenamiento que les dé mayor capacidad resolutiva para las principales patologías que se presentan en la comunidad. Así mismo, deberá mejorarse la dotación de transporte y la red de comunicaciones para estos promotores.

Finalmente, es necesario que las comunidades aclaren qué tipo de relación han establecido con diferentes aspectos de la cultura occidental y en qué situación de aculturación se encuentran, de manera que puedan tener mayor claridad con respecto al tipo de desarrollo que quieren tener. Todo este proceso, largo y complicado, también implica que las personas que trabajan con comunidades indígenas tengan un conocimiento claro con respecto a su propia cultura.

\section{Bibliografía}

1. Arango R, Sánchez E. Los pueblos indígenas de Colombia. Depatamento Nacional de Planeación. Bogotá, 1989.

2. Baruzzi RG, Marcopito LF, Serra FA, et al. The Kren-Akorore: a recently contacted indigenous tribe, disease in tribal societies, CIBA Foundation Symposium 49, Elsevier Amsterdam, 1979; 79.

3. Kroeger A, Barbira-Freedmann F. Cambio cultural y salud. Ed. Mundo Shuar. Quito, 1984.

4. Kleinmann A. Patients and healers in the context of culture. University of California Press Ltd. London, 1980.

5. Bannermann R, Burton J, Wen-Chieh Ch. Traditional medicine and health care coverage, WHO. Geneva, 1983.

6. Pinzón C, Suárez R. Las mujeres lechuza: historia, cuerpo y brujería en Boyacá. Ican/Colcultura y Cerec. Santafé de Bogotá, 1992.

7. Ministerio de Salud, Decreto Número 1811 de 1990. 